\title{
Low-grade sulfadoxine-pyrimethamine resistance in Plasmodium falciparum parasites from Lubango, Angola
}

\author{
Elsa P. S. Kaingona-Daniel 1,2,3,7, Larissa Rodrigues Gomes 1,2,7, Bianca E. Gama 1,4, \\ Natália K. Almeida-de-Oliveira ${ }^{1,2,7}$, Filomeno Fortes ${ }^{5,7}$, Didier Ménard ${ }^{6}$, Cláudio Tadeu Daniel-Ribeiro 1,2,7 \\ and Maria de Fátima Ferreira-da-Cruz ${ }^{1,2,7^{*}}$
}

\begin{abstract}
Background: Malaria is a major parasitic disease, affecting millions of people in endemic areas. Plasmodium falciparum parasites are responsible for the most severe cases and its resistance to anti-malarial drugs is notorious. This is a possible obstacle to the effectiveness of intermittent preventive treatment (IPT) based on sulfadoxine-pyrimethamine (SP) cures administrated to pregnant women (IPTp) during their pregnancy. As this intervention is recommended in Angola since 2006, it has assessed, in this country, the molecular profiles in P. falciparum dhfr and dhps, two polymorphic genes associated to pyrimethamine and sulfadoxine resistance, respectively.

Methods: Blood samples from 52 falciparum patients were collected in Lubango, Angola and pfdhfr and pfdhps polymorphisms were analysed using nested-PCR and DNA sequencing.

Results: In the pfdhfr gene, the $108 \mathbf{N}$ mutation was almost fixed (98\%), followed by $59 \mathbf{R}(63 \%), 51 \mathbf{I}(46 \%), 50 \mathbf{R}$ and $164 \mathbf{L}(2 \%$, respectively). No $16 \mathbf{V} / \mathbf{S}$ mutations were found. The most common double mutant genotype was CNRN $(59+108 ; 46 \%)$, followed by CICN $(51+108 ; 29 \%)$ whereas IRN $(51+59+108 ; 15 \%)$, CNRNVL $(59+108+164$; $2 \%)$ and $\mathbf{R I C N}(50+51+108 ; 2 \%)$ triple mutant genotypes were detected. Investigations of the pfdhps gene showed that the $437 \mathbf{G}$ mutation was the most prevalent ( $97 \%)$. Only two and one samples disclosed the $540 \mathbf{E}$ (7\%) and the 436A (3\%), respectively. Single mutant SGKAA (437; $86 \%)$ was higher than SGEAA (437 + 540; $7 \%$ ) or AGKAA $(436+437 ; 3 \%)$ double mutants genotypes. No polymorphism was detected at codons $581 \mathbf{G}$ and $613 \mathbf{T} / \mathbf{S}$. Combining pfdhfr and pfdhps alleles two triple mutant haplotypes (double mutant in dhfr and single mutant in dhps) were observed: the ACICNVI/S $\underline{\mathbf{G} K A A}$ in $14(56 \%)$ samples and the ACNRNVI/SGKAA in five (20\%) samples. One quadruple mutant haplotype was detected (ACIRNVI/SGKAA) in six (24\%) P. falciparum samples. No quintuple pfdhfrpfdhps mutant was noted.

Conclusion: pfdhfr and pfdhps gene mutations in isolates from Lubango are suggestive of a low-grade SP resistance and IPT for pregnant women and infant based on SP treatment could be effective. Routine molecular studies targeting polymorphism in these two genes need to be routinely conducted at country level.
\end{abstract}

Keywords: Malaria, P. falciparum, pfdhfr, pfdhps, Sulfadoxine, Pyrimethamine, Angola

\section{Background}

Plasmodium falciparum malaria is the one of the major

*Correspondence: mffcruz@ioc.fiocruz.br; mffcruz28@gmail.com

1 Laboratório de Pesquisa em Malária—Instituto Oswaldo Cruz, Fundação

Oswaldo Cruz (Fiocruz), Rio de Janeiro, Brazil

Full list of author information is available at the end of the article cause of morbidity and mortality in sub-Saharan Africa, including Angola, where three million clinical cases and 6000 deaths occurred in 2015. The deaths reported in Angola territory, accounting for $35 \%$ in children under five years old and $25 \%$ of the maternal deaths (Angolan National Malaria Control Programme, 2015). 
In endemic malaria communities pregnant women and infants are more vulnerable to malaria episodes [1]. The World Health Organization strategy to protect mothers during their pregnancy and consequences in newborns such as low weight includes the implementation of intermittent preventive treatment in pregnant women (IPTp) [2]. This treatment was introduced in Angola in 2006, using the sulfadoxine-pyrimethamine (SP) at the second trimester of pregnancy. However, the emergence of $P$. falciparum resistances to both sulfadoxine and pyrimethamine can jeopardize these strategies.

Sulfadoxine-pyrimethamine resistant parasites are frequent in Southeast Asia and are becoming more and more prevalent in several East African countries [3]. SP acts by inhibiting the $P$. falciparum dihydrofolate reductase $(d h f r)$ and dihydropteroate synthetase (dhps), two fundamental enzymes involved in the folate biosynthesis pathway [4]. Single nucleotide polymorphisms (SNPs) leading non-synonymous mutations in these two genes have been shown to be associated with pyrimethamine (dhfr) and sulfadoxine (dhps) resistances. The Angolan National Malaria Control Programme intends to introduce IPT in infants, as has already been done in other sub-Saharan Africa countries. To inform current policy makers when recommending the use of SP in IPT, $d h f r$ and dhps molecular markers are used to differentiate high and low grade SP resistance areas and to define its geographical distribution [5].

Malaria is endemic throughout Angola, but the transmission patterns is heterogenous varying from intense transmission and hyperendemicity in northern, mesoendemic stable in the centre, to seasonal or epidemic unstable malaria in the southern part of the country [6]. No study for investigating pfdhfr and pfdhps mutations was performed in southern part of Angola where Lubango is located. The great majority of previous studies were conducted at the northern in Luanda, [7, 8]; Uige [9, 10], Cabinda, Kwanza Norte and Malanje [10], followed by those at the Central Angola in Benguela [11] and Huambo [10].

The objective of this study was to investigate the polymorphism at codons A16V/S, C50R, N51I, C59R, S108N, V140L and $1164 \mathrm{~L}$ in the pfdhfr gene and at codons S436A/F/C, A437G, K540E, A581G and A613T/S in the pfdhps gene, in order to provide baseline data regarding the proportion of $P$. falciparum pfdhfr and pfdhps mutations, before SP-IPTi introduction in Lubango, an Angolan malaria stable transmission region.

\section{Methods}

\section{Study site, blood samples and DNA extraction}

Blood samples were collected in 2011 from 52 patients presenting falciparum malaria at the Central Hospital in
Lubango, located in Huila Province, South of Luanda, Angola capital. Lubango is located in southern Angola, with an area of 79,022 $\mathrm{Km}^{2}, 1790 \mathrm{~m}$ above sea level, with an estimated population of $1,414,115$. Malaria transmission occurs throughout the year, with peaks during rainy season, between March and May. Malaria is mesoendemic and $P$. falciparum is the predominant malaria species. Inclusion criteria comprised individuals with $P$. falciparum aging over 12 years and no evidence of complicated malaria. The Central Hospital is the main health facility in Lubango and it is a reference for malaria in the area. The malaria diagnosis was performed by thick blood smear and nested-PCR, as previous described [12]. All malaria cases were treated following the Angolan malaria treatment policy. After obtaining informed consent, venous blood collection was performed according to protocols approved by the National Institute of Public Health and the Ethical Committee of Angola and Fiocruz, Brazil (\#372/07). The samples were cryopreserved and stored at $-20{ }^{\circ} \mathrm{C}$ until DNA extraction. Genomic DNA was extracted from $1 \mathrm{~mL}$ whole blood using the QIAamp Midi columns (Qiagen), as described by the manufacturer.

\section{Nested polymerase chain reaction (PCR) and electrophoresis}

$p f d h f r$ and $p f d h p s$ genes were amplified by nested-PCR approach using two gene-specific primers (external and internal) as already reported [13]. The nested-PCRs were performed to detect the presence of mutations at codons: A16V/S, C50R, N51I, C59R, S108N, V140L and I164L in the pfdhfr gene and at codons S436A/F/C, A437G, $\mathrm{K} 540 \mathrm{E}, \mathrm{A} 581 \mathrm{G}$ and $\mathrm{A} 613 \mathrm{~T} / \mathrm{S}$ in the $p f d h p s$ gene. The choice of these codons was based on the pioneer study of Pearson and colleagues [13].

\section{DNA sequencing and SNP polymorphisms detection}

After purification of the amplicons using the Wizard SV Gel and PCR Clean-Up System (Promega), PCR products were sequenced using Big Dye ${ }^{\circledR}$ Terminator Cycle Sequencing Ready Reaction version 3.1 (Applied Biosystems) and ABI PRISM 3730 DNA Analyzer [14] at the Genomic Platform/PDTIS/Fiocruz. The sequences of the amplicons were aligned with the wild-type 3D7 strain for pfdhfr (GenBank accession number XM_001351443) and for pfdhfr (GenBank accession number Z30654). The presence of SNPs was confirmed by reading both the forward and the reverse strands using the free software Bioedit Sequence Alignment Editor Version 7.2.5. Parasites with mixed alleles (in which both wild-type and mutant alleles were present) were considered mutants for estimation of the prevalence of the SNPs. Haplotypes for drug resistance markers were reconstructed from the full 
sequence presenting an unambiguous single allele signal at all positions. Statistical significance of differences between the haplotypes was assessed using Fisher's tests and a $p$ value $<0.05$ was considered significant.

\section{Results}

Among the 52 successfully sequenced samples for $d h f r$, 51 (98\%) showed non-synonymous mutations. The most frequent mutation was $108 \mathrm{~N}(51 / 52$; $98 \%, 95 \% \mathrm{CI})$ followed by 59R (33/52; $63 \%, 95 \% \mathrm{CI})$ and $51 \mathrm{I}(24 / 52 ; 46 \%$, $95 \% \mathrm{CI})$. The $50 \mathrm{R}$ and $164 \mathrm{~L}$ mutations were detected at the same frequencies $(1 / 52 ; 2 \%, 95 \% \mathrm{CI})$ and no mutations were found at codons $16 \mathrm{~V} / \mathrm{S}$ and $140 \mathrm{~L}$ (Table 1).

Double mutants $(51 \mathbf{I} / 108 \mathbf{N}, 59 \mathbf{R} / 108 \mathrm{~N})$ were observed in 39 isolates $(76.5 \% ; 39 / 51)$ and triple mutants $(51 \mathbf{I} / 59 \mathbf{R} / 108 \mathbf{N}, \quad 50 \mathbf{R} / 51 \mathbf{I} / 108 \mathbf{N}, \quad 59 \mathbf{R} / 108 \mathbf{N} / 164 \mathbf{L})$ in ten $(19.5 \%$; 10/51) P. falciparum samples. The $108 \mathrm{~N}$ single mutant was found in only two $(4 \% ; 2 / 51)$ isolates (Table 1). In samples presenting dhfr double mutation, the CNRN $(59+108)$ allele was the most frequent $(24 / 46 \%)$ followed by CICN $(51+108)(15 / 29 \%)$. This contrasts with the frequency of the $d h f r$ triple mutant alleles which were detected at lower rates: IRN $(51+59+108), \mathbf{C N} \underline{\mathbf{R N V}} \underline{\mathbf{L}}(59+108+164)$ and $\underline{\mathbf{R I C N}}$ $(50+51+108)(8(15 \%), 1(2 \%)$ and $1(2 \%)$, respectively). The wild-type ACNCSVI was presented in only one isolate (Tables 2 and 3 ).

Of the 52 samples tested, 29 (56\%) were successfully amplified for the dhps gene. Among them 28 (97\%) presented a mutation at codons S436A, A437G and K540E. The 437G mutant allele was highly prevalent (97\%), while the $540 \mathrm{E}(7 \%)$ and $436 \mathrm{~A}$ (3\%) were observed in lower frequencies. No $581 \mathbf{G}$ and $613 \mathrm{~T} / \mathrm{S}$ alleles were observed (Table 1). The frequency of the single mutant SGKAA (437) (25/86 \%) was higher than double mutants SGEAA $(437+540)(2 / 7 \%)$ or AGKAA $(436+437)$ $(1 / 3 \%)(\mathrm{p}<0.05)$. The dhps wild-type SAKAA was rare and observed in only one (3\%) P. falciparum sample (Tables 2 and 3 ).

Table 1 Plasmodium falciparum dhfr and dhps mutated codons in parasites from Lubango, Angola

\begin{tabular}{lcc}
\hline Gene & SNPs & $\begin{array}{c}\text { Prevalence } \\
\mathbf{N}(\%)\end{array}$ \\
\hline$d h f r(\mathrm{n}=52)$ & $50 \mathbf{R}$ & $1(2)$ \\
$95 \% \mathrm{Cl}$ & $51 \mathbf{I}$ & $24(46)$ \\
& $59 \mathbf{R}$ & $33(63)$ \\
& $108 \mathbf{N}$ & $51(98)$ \\
& $164 \mathbf{L}$ & $1(2)$ \\
dhps $(n=29)$ & $436 \mathbf{A}$ & $1(3)$ \\
$95 \% \mathrm{Cl}$ & $437 \mathbf{G}$ & $28(97)$ \\
& $540 \mathbf{E}$ & $2(7)$ \\
\hline
\end{tabular}

Table 2 Prevalence of $P$. falciparum $d h f r$ and dhps mutants in parasites from Lubango, Angola

\begin{tabular}{|c|c|c|c|}
\hline Gene & Mutation & SNPs & $\begin{array}{l}\text { Prevalence } \\
\text { N (\%) }\end{array}$ \\
\hline \multirow[t]{6}{*}{$d h f r n=51$} & Single & S108N & $2(4)$ \\
\hline & Double & N51I/S108N & $15(29)$ \\
\hline & & C59R/S108N & $24(47)$ \\
\hline & Triple & N51I/C59R/S108N & $8(16)$ \\
\hline & & C50R/N51I/S108N & $1(2)$ \\
\hline & & C59R/S108N//164L & $1(2)$ \\
\hline \multirow[t]{3}{*}{ dhps $n=28$} & Single & $A 437 \mathbf{G}$ & $25(89)$ \\
\hline & Double & $\mathrm{A} 437 \mathbf{G} / \mathrm{K} 540 \mathbf{E}$ & $2(7)$ \\
\hline & & S436A/A437G & $1(4)$ \\
\hline
\end{tabular}

Table 3 Deduced haplotype profiles for dhfr and $\operatorname{dhps} P$. falciparum parasites from Lubango, Angola

\begin{tabular}{llrr}
\hline Gene & Haplotypes & N & $\%$ \\
\hline dhfr $(\mathrm{n}=52)$ & CNRN & 24 & 46 \\
& CICN & 15 & 29 \\
& CIRN & 8 & 15 \\
& CNCN & 2 & 4 \\
& CNRNVL & 1 & 2 \\
& RICN & 1 & 2 \\
dhps $(\mathrm{n}=29)$ & ACNCSVI & 1 & 26 \\
& SGKAA & 25 & 7 \\
& SGEAA & 2 & 3 \\
& AGKAA & 1 & 3 \\
\hline
\end{tabular}

Combining pfdhfr and pfdhps alleles, not all isolates had an interpretable haplotype (Table 4). The PCR amplification failure rate of the dhps gene in Lubango samples might be somehow attributed to primer mismatched due to unknown polymorphisms in target sequences, because in all the 52 samples amplification was achieved when primers strictly developed to amplify conserved sequences for diagnosis purposes were tested [12]. The number of samples herein considered was based on amplification of both PCRs just to avoid the identification of incomplete haplotypes.

Two triple mutant haplotypes (double mutant in $d h f r$ and single mutant in dhps) were observed: the ACIC-

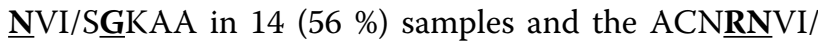
SGKAA in five $(20 \%)$ samples. One quadruple mutant haplotype was detected (ACIRNVI/SGKAA) in six (24\%) $P$. falciparum samples (Table 4). The high-grade SP resistant haplotypes (quintuple, sextuple and septuple $p f d h f r /$ pfdhps) [3] were not seen among these isolates. 


\section{Discussion}

At present, SP continues to be used in IPTp and IPTi strategies, which are likely to provide sub-optimal effect. Therefore, monitoring the spread of SP resistance using $d h f r$ and dhps as molecular markers are strongly recommended [15]. This study was performed with P. falciparum samples from Lubango, an Angola region with unstable meso-endemic malaria transmission. The vast majority of tested isolates (98\%) presented at least one mutation in the gene encoding enzymes associated with pyrimethamine resistance.

High prevalence of $108 \mathbf{N}$ mutation (considered as the initial mutation occurring in $d h f r$ multiple mutants) was detected, similar to observations already done in several Angolan regions $[8,10,11]$ and in other African countries [16-26]. However, contrary to various Angolan localities, the $59 \mathbf{R}$ was the second more frequent $d h f r$ mutation in Lubango, instead of the 51I [8-11]. In addition, it is worth to noting that the 164L pfdhfr SNP was also detected for the first time in Angola. This mutation is rare in Africa [5] but its presence in association with $108 \mathbf{N}+51 \mathbf{I}$ and/or $59 \mathbf{R}$ [27] as well as with the $d h f r+d h p s$ quintuple mutant [5], confers high levels of in vivo and in vitro resistance that could render SP totally ineffective. The 164L mutation was also combined to cycloguanil resistance [28] and is commonly found in $P$. falciparum samples from Southwest Asia [29, 30] and South America [31]. The possible reason for the detection of mutant 164L in Lubango (north of Angola) and not in other correlate Angolan studies (North and Central Angola) is, probably, due to the greater proximity of Lubango with Zambia, where the presence of quintuple mutants is greater than $50 \%$ [3].

From previous reports, it was known that the triple African mutant (N51I + C59R + S108N) shares a common ancestry with resistant $d h f r$ from Southeast Asia [32, 33]; this mutant emerged in Asia and was introduced later in Africa, including Angola [19, 20, 33-36]. With respect to $d h f r$ mutation $50 \mathrm{R}$, another non-frequent $d h f r$ mutation in Africa, it was also identified in this study. This low-grade pyrimethamine resistant mutant [27] was first described as specific of South American isolates [27] but, besides South America, it has already been described

Table 4 Combined molecular dhfr and dhps gene profiles in $\mathbf{2 5}$ samples of $P$. falciparum from Lubango, Angola

\begin{tabular}{|c|c|c|c|}
\hline \multirow{2}{*}{$\begin{array}{l}\text { Genes } \\
d h f r+d h p s\end{array}$} & \multirow[t]{2}{*}{ Haplotypes } & \multicolumn{2}{|c|}{ Mutated } \\
\hline & & $\mathrm{N} / \%$ & Type \\
\hline $511 / 108 N+437 G$ & $A C \underline{I} C \underline{\mathbf{N}} \mathrm{VI}+S \underline{\mathbf{G}} \mathrm{KAA}$ & $14 / 56$ & Double and single \\
\hline $59 R / 108 N+437 G$ & $A C N \underline{\mathbf{R N}} \mathrm{V} I+S \underline{\mathbf{G}} K A A$ & $5 / 20$ & Double and single \\
\hline $511 / 59 R / 108 N+437 G$ & $\mathrm{ACI \mathbf {RN }} V \mid+S \underline{\mathbf{G}} K A A$ & $6 / 24$ & Triple and single \\
\hline
\end{tabular}

in Africa in isolates from Kenya [35], Central African Republic [37] and Angola [8].

In relation to dhps gene, the $437 \mathbf{G}$ mutation was the most common, reaching almost fixation, similarly to other Angolan localities [8, 10, 11] and other African countries $[16,20,23,26]$, especially in western and Central Africa.

The dhps 540E mutation, less commonly observed in western Africa, was also identified in this study, confirming previous data from Luanda [7, 8], Cabinda, Huige, Kwanza Norte, Malange and Huambo [10]. However, the spatial distribution of this allele in Angolan provinces seems heterogeneous and even in the same province its frequency can vary overtime $[9,10]$. Thus, screening for dhps 540E, a surrogate for the quintuple mutant, is a priority in West Africa where it is comparatively rare [3].

The combination of $437 \mathbf{G}$ with the triple mutant $51 \mathbf{I}+59 \mathbf{R}+108 \mathrm{~N}$ which is considered to be associated with SP treatment failure [38-40], was detected in $33 \%$ of Lubango samples. However, in vitro studies showed that the single 437G mutant has a lesser degree of tolerance to sulfadoxine than the $437 \mathbf{G}+540 \mathrm{E}$ double mutant. Thus, triple mutant $51 \mathbf{I}+59 \mathbf{R}+108 \mathrm{~N}$ associated with single 437G mutant could have a less detrimental effect on SPIPT than the associated $437 \mathbf{G}+540 \mathrm{E}$ mutations-the sustained protective efficacy of SP-IPTi in western and Central Africa reinforce these points [41].

Despite the presence of parasites with triple $d h f r$ mutant at positions 51I, 59R and 108N (15\%) and double dhps mutant at positions $437 \mathbf{G}+540 \mathbf{E}(7 \%)$, the quintuple mutant $(51 \mathbf{I}+59 \mathbf{R}+108 \mathbf{N}+437 \mathbf{G}+540 \mathbf{E})$, considered a significant predictor of SP treatment failure [42] was not detected in the studied isolates from Lubango. To date, this quintuple mutant was not observed in central and northern areas of Angola [7-11]. Besides Lubango, similar low frequencies of double $437 \mathbf{G}+540 \mathbf{E}$ pfdhps mutant were described in Huambo (12.5\%) [10], Benguela (8 \%) [10], Cabinda (5.3 \%) [10], Malanje (3.1\%) [10], Luanda (3 \%) [8], Kwanza Norte (2.1 \%) [10] and Uige (1.9\%) [10], contrasting with the higher frequencies of this mutant detected in Eastern Africa: Kenya (86 \%), Tanzania (90\%), Malawi (95\%) and Uganda (95\%). With the exception of Malanje (3.9\%), the frequencies of the triple $51 \mathbf{I}+59 \mathbf{R}+108 \mathbf{N} d h f r$ mutant in Angolan provinces, including Lubango, were higher than the double $437 \mathbf{G}+540 \mathbf{E}$ pfdhps mutant but, East African countries presented higher prevalence rates [43].

These findings are expected because East African countries presented a high rate of SP treatment failure of falciparum malaria together with the increased presence of the quintuple mutant considered the most specific molecular marker for SP $[44,45]$. 
Besides quintuple mutants, the concept of "super resistant genotypes" is emerging which further raise the threshold of drug tolerance in parasites comprising additional mutations in combination with N51I + C59R + S108N and $\mathrm{A} 437 \mathbf{G}+\mathrm{K} 540 \mathrm{E}$ [3]. The additional mutations are dhfr I164L (found in one sample; $2 \%$ ) and dhps A581G and A613T/S, which were not found in this study. Although, these mutations are rare, their impact on SP-IPTi or SPIPTp efficacy is considered highly detrimental [5]. When the quadruple mutant N51I + C59R + S108N + A437G occur in the absence of $540 \mathrm{E}$, these parasite populations are classified as partially resistant genotypes, and its resistance levels are not expected to be as high [3]. The efficacy of IPTp in western Africa, where N51I + C59R + S108N and A437G exist in combination with 581G but not with $540 \mathrm{E}$ supports the view that these parasites should be regarded differently [3]. In this case, molecular markers of super resistance may be an important warning about failing efficacy of IPTp.

Many studies suggested that IPTp with SP remains effective, or at least it is not associated with harm, in areas with high prevalence of $P$. falciparum quintuple mutant parasites $[46,47]$. There is not enough evidence yet to establish a threshold prevalence of the classical $d h f r+d f p s$ quintuple mutant, or even of the dhps 581, dhps 540 or dhfr 164 point mutations above which there is a clear loss of IPTp-SP effectiveness. Therefore, the significance of the additional mutation at codon 540 needs further investigation.

\section{Conclusion}

In view of the data reported here and elsewhere, it was conclude that, although the number of samples available for the present study was limited, $p f d h f r$ and $p f d h p s$ gene mutations in isolates from Lubango are suggestive of a low-grade SP resistance and their presences highlight the importance of molecular markers screening to monitor the evolution of $P$. falciparum SP resistance in Angola.

\section{Authors' contributions \\ MFFC carried out the study and the manuscript. EPSKD performed and ana- lysed DNA sequencing and drafted the manuscript. LRG performed statistical analysis and edited the manuscript. BEG reviewed the DNA sequences. DM and CTDR participated in the discussions and reviewed the final manuscript. NKAO performed DNA extraction and PCRs. FF recruited the patients. All authors read and approved the final manuscript.}

\footnotetext{
Author details

' Laboratório de Pesquisa em Malária—Instituto Oswaldo Cruz, Fundação Oswaldo Cruz (Fiocruz), Rio de Janeiro, Brazil. ${ }^{2}$ Centro de Pesquisa, Diagnóstico e Treinamento em Malária (CPD-Mal) Fiocruz, Rio de Janeiro, Brazil. ${ }^{3}$ Hospital Central Dr. António Agostinho Neto, Lubango, Angola. ${ }^{4}$ Laboratory of Oncovirology, Instituto Nacional de Câncer, Rio de Janeiro, Brazil. ${ }^{5}$ Angolan National Malaria Control Programme, National Institute of Public Health, Luanda, Angola. ${ }^{6}$ Malaria Molecular Epidemiology Unit, Institut Pasteur in Cambodia, Phnom Penh, Cambodia. ${ }^{7}$ Health Progress and Investigation Network of the Portuguese-Speaking Countries Community (RIDESMal/CPLP), Lisbon, Portugal.
}

\section{Acknowledgements}

We thank all the patients who voluntarily agreed in participate in this work, as well as the Angolan Ministry of Health and FESA (Eduardo dos Santos Foundation). CTDR and MFFC are recipients of a Research Productivity Fellowship from the National Brazilian Council for Scientific and Technological Development (CNPq) and from the Foundation for Research Support in the State of Rio de Janeiro (Faperj) as "Cientistas do Nosso Estado". The sponsors had no role in the collection, analyses and interpretation of data, in the writing of the manuscript and in decision to submit the manuscript for publication.

\section{Competing interests}

The authors declare that they have no competing interests.

Received: 24 March 2016 Accepted: 24 May 2016

Published online: 07 June 2016

\section{References}

1. Tutu EO, Lawson B, Browne E. The effectiveness and perception of the use of sulphadoxine-pyrimethamine in intermittent preventive treatment of malaria in pregnancy programme in Offinso district of Ashanti region, Ghana. Malar J. 2011;10:385.

2. WHO. A strategic framework for malaria prevention and control during pregnancy in the African region, World Health Organization. 2004.

3. Naidoo I, Roper C. Mapping 'partially resistant,' 'fully resistant' and 'super resistant' malaria. Trends Parasitol. 2013;10:505-15. doi:10.1016/j. pt.2013.08.002.

4. Brooks DR, Wang P, Read M, Watkins WM, Sims PF, Hyde JE. Sequence variation of the hydroxymethyldihydropterin pyrophosphokinase: dihydropteroate synthase gene in lines of the human malaria parasite, Plasmodium falciparum, with differing resistance to sulfadoxine. Eur J Biochem. 1994;2:397-405.

5. Venkatesan M, Alifrangis M, Roper C, Plowe CV. Monitoring antifolate resistance in intermittent preventive therapy for malaria. Trends Parasitol. 2013;10:497-504. doi:10.1016/j.pt.2013.07.008.

6. Angola malaria indicator survey 2011. final report. http://dhsprogram. com/pubs/pdf/MIS11/MIS11.pdf.

7. Figueiredo P, Benchimol C, Lopes D, Bernardino L, Rosario VE, Varandas $L$, et al. Prevalence of pfmdr1, pfcrt, pfdhfr and pfdhps mutations associated with drug resistance, in Luanda, Angola. Malar J. 2008;7:236. doi:10.1186/1475-2875-7-236

8. Gama BE, Pereira-Carvalho GA, Lutucuta Kosi FJ, Almeida de Oliveira NK, Fortes F, Rosenthal PJ, et al. Molecular markers of antifolate resistance in Plasmodium falciparum isolates from Luanda, Angola. Malar J. 2011;10:248. doi:10.1186/1475-2875-10-248.

9. Menegon M, Pearce RJ, Inojosa WO, Pisani V, Abel PM, Matondo A, Bisoffi Z, Majori G, et al. Monitoring for multidrug-resistant Plasmodium falciparum isolates and analysis of pyrimethamine resistance evolution in Uige province, Angola. Trop Med Int Health. 2009;14:1251-7. doi:10.111 1/j.1365-3156.2009.02369.

10. Fortes F, Dimbu R, Figueiredo P, Neto Z, do Rosário VE, Lopes D. Evaluation of prevalences of pfdhfr and pfdhps mutations in Angola. Malar J. 2011;10:22. doi:10.1186/1475-2875-10-22.

11. Ngane FV, Allico Djaman J, Culeux C, Piette N, Carnevale P, Besnard P, et al. Molecular epidemiology of drug-resistant Plasmodium falciparum in Benguela province, Angola. Malar J. 2015;14:113. doi:10.1186/ s12936-015-0634-2.

12. Zalis MG, Ferreira-da-Cruz MF, Balthazar-Guedes HC, Banic DM, Alecrim W, Souza JM, et al. Malaria diagnosis: standardization of a polymerase chain reaction for the detection of Plasmodium falciparum parasites in individuals with low-grade parasitemia. Parasitol Res. 1996;82:612-6.

13. Pearce RJ, Drakeley C, Chandramohan D, Mosha F, Roper C. Molecular determination of point mutation haplotypes in the dihydrofolate reductase and dihydropteroato synthase of Plasmodium falciparum in three districts of northern Tanzania. Antimicrob Agents Chemother. 2003;47:1347-54. doi:10.1128/AAC.47.4.

14. Otto TD, Vasconcellos EA, Gomes LH, Moreira AS, Degrave WM, Mendonça-Lima L, et al. ChromaPipe: a pipeline for analysis, quality 
control and management for a DNA sequencing facility. Genet Mol Res. 2008;23:861-71.

15. Alifrangis M, Nag S, Schousboe ML, Ishengoma D, Lusingu J, Pota H. Independent origin of Plasmodium falciparum antifolate super-resistance, Uganda, Tanzania and Ethiopia. Emerg Infect Dis. 2014;20:1280-6.

16. Gebru-Woldearegai T, Hailu A, Grobusch MP, Kun JF. Molecular surveillance of mutations in dihydrofolate reductase and dihydropteroate synthase genes of Plasmodium falciparum in Ethiopia. Am J Trop Med Hyg. 2005;6:1131-4.

17. Schunk M, Kumma WP, Miranda IB, Osman ME, Roewer S, Alano A, Löscher T, et al. High prevalence of drug-resistance mutations in Plasmodium falciparum and Plasmodium vivax in southern Ethiopia. Malar J. 2006:5:54. doi:10.1186/1475-2875-5-54.

18. Nkhoma S, Molyneux M, Ward S. Molecular surveillance for drug-resistant Plasmodium falciparum malaria in Malawi. Acta Trop. 2007;102:138-42. doi:10.1016/j.actatropica.2007.04.006.

19. Certain LK, Briceno M, Kiara SM. Characteristics of Plasmodium falciparum dhfr haplotypes that confer pyrimethamine resistance, Kilifi, Kenya, 1987-2006. J Infect Dis. 2008;197:1743-51.

20. Lynch C, Pearce R, Pota H. Emergence of a dhfr mutation conferring high-level drug resistance in Plasmodium falciparum populations from Southwest Uganda. J Infect Dis. 2008;197:1598-604. doi:10.1086/588198.

21. Zhong D, Afrane Y, Githeko A, Cui L, Menge DM, Yan G. Molecular epidemiology of drug-resistant malaria in western Kenya highlands. BMC Infect Dis. 2008;31:105. doi:10.1186/1471-2334-8-105.

22. Alifrangis M, Lusingu JP, Mmbando B, Dalgaard MB, Vestergaard LS, Ishengoma D. Five-year surveillance of molecular markers of Plasmodium falciparum antimalarial drug resistance in Korogwe District, Tanzania: accumulation of the $581 \mathrm{G}$ mutation in the P. falciparum dihydropteroate synthase gene. Am J Trop Med Hyg. 2009;4:523-7.

23. Bonizzoni M, Afrane Y, Baliraine FN, Amenya DA, Githeko AK, Yan G. Genetic structure of Plasmodium falciparum populations between lowland and highland sites and antimalarial drug resistance in Western Kenya. Infect Genet Evol. 2009;9:806-12. doi:10.1016/j. meegid.2009.04.015.

24. Oesterholt MJ, Alifrangis M, Sutherland CJ, Omar SA, Sawa P, Howitt $C$, et al. Submicroscopic gametocytes and the transmission of antifolate-resistant Plasmodium falciparum in Western Kenya. PLoS One. 2009;4:4364. doi:10.1371/journal.pone.0004364.

25. Karema C, Imwong M, Fanello Cl, Stepniewska K, Uwimana A, Nakeesathit $\mathrm{S}$, et al. Molecular correlates of high-level antifolate resistance in Rwandan children with Plasmodium falciparum malaria. Antimicrob Agents Chemother. 2010;54:477-83. doi:10.1128/AAC.00498-09.

26. Salgueiro P, Vicente JL, Ferreira C, Teófilo V, Galvão A, do Rosário VE, et al. Tracing the origins and signatures of selection of antifolate resistance in island populations of Plasmodium falciparum. BMC Infect Dis. 2010;10:163. doi:10.1186/1471-2334-10-163.

27. Cortese JF, Caraballo A, Contreras CE, Plowe CV. Origin and dissemination of Plasmodium falciparum drug-resistance mutations in South America. J Infect Dis. 2002;186:999-1006.

28. Basco LK, Pécoulas PE, Wilson CM, Le Bras J, Mazabrald A. Point mutations in the dihydrofolate reductase-thymidylate synthase gene and pyrimethamine and cycloguanil resistance in Plasmodium falciparum. Mol Biochem Parasitol. 1995;69:135-8.

29. Foote SJ, Gálatas D, Cowman AF. Amino acids in the dihydrofolate reductase-thymidylate synthase gene of Plasmodium falciparum involved in cycloguanil resistance differ from those involved in pyrimethamine resistance. Proc Nat Acad Sci USA. 1990;87:3014-7.

30. Peterson DS, Milhous WK, Wellems TE. Molecular basis of differential resistance to cycloguanil and pyrimethamine in Plasmodium falciparum malaria. Proc Nat Acad Sci USA. 1990;87:3018-22.

31. Vasconcelos KF, Plowe CV, Fontes CJ, Kyle D, Wirth DF, Pereira da Silva LH. Mutations in Plasmodium falciparum dihydrofolate reductase and dihydropteroate synthase of isolates from the Amazon region of Brazil. Mem Inst Oswaldo Cruz. 2000;95:721-8.

32. Roper C, Pearce R, Nair S, Sharp B, Nosten F, Anderson T. Intercontinental spread of pyrimethamine-resistant malaria. Science. 2004;305:1124. doi:10.1126/science.1098876.

33. Maiga O, Djimde AA, Hubert V. A shared Asian origin of the triple-mutant dhfr allele in Plasmodium falciparum from sites across Africa. J Infect Dis. 2007;196:165-72.

34. Roper C, Pearce R, Bredenkamp B. Antifolate antimalarial resistance in southeast Africa. Lancet. 2003;361:1174-81.

35. McCollum AM, Poe AC, Hamel M. Antifolate resistance in Plasmodium falciparum: multiple origins and identification of novel dhfr alleles. J Infect Dis. 2006;194:189-97.

36. Ndiaye D, Daily JP, Sarr O. Defining the origin of Plasmodium falciparum resistant dhfr isolates in Senegal. Acta Trop. 2006;99:106-11. doi:10.1016/j. actatropica.2006.07.002.

37. Menard D, Djalle D, Yapou F, Manirakiza A, Talarmin A. Frequency distribution of antimalarial drug-resistant alleles among isolates of Plasmodium falciparum in Bangi, Central African Republic. Am J Trop Med Hyg. 2006:3:2005-10.

38. Mockenhaupt FP, Teun BJ, Eggelte TA, Schreiber J, Ehrhardt S, Wassilew N, Otchwemah RN, Sauerwein RW, Bienzle U. Plasmodium falciparum dhfr but not dhps mutations associated with sulphadoxine-pyrimethamine treatment failure and gametocyte carriage in Northern Ghana. Trop Med Int Health. 2005;10:901-8.

39. Kun JF, Lehman LG, Lell B, Schimidt-Ott R, Kremsner PG. Low-dose treatment with sulfadoxine-pyrimethamine combinations select for drugresistant Plasmodium falciparum strains. Antimicrob Agents Chemother. 1999:9:2205-8.

40. Ndounga M, Tahar R, Basco LK, Casimiro PN, Malonga DA, Ntoumi F. Therapeutic efficacy of sulfadoxine-pyrimethamine and the prevalence of molecular markers of resistance in under 5-year olds in Brazzaville. Congo. Trop Med Int Health. 2007:12:1164-71.

41. Griffin JT, Cairns M, Ghani AC, Roper C, Schellenberg D, Carneiro I, et al. Protective efficacy of intermittent preventive treatment of malaria in infants (IPTi) using sulfadoxine-pyrimethamine and parasite resistance. PLoS One. 2010:5:e12618.

42. Picot S, Olliaro, de Monbrinson F, Bienvenu AL, Price RN, Ringwald P. A systematic review and meta-analysis of evidence for correlation between molecular markers of parasite resistance and treatment outcome in falciparum malaria. Malar J. 2009;4:89. doi:10.1 186/1475-2875-89.

43. Sridaran S, McClintock SK, Syphard LM, Herman KM, Barnwell JW, Udhayakumar $\mathrm{V}$. Anti-folate drug resistance in Africa: meta-analysis of reported dihydrofolate reductase (dhfr) and dihydropteroate synthase (dhps) mutant genotype frequencies in African Plasmodium falciparum parasite populations. Malar J. 2010;9:247. doi:10.1186/1475-2875-9-247.

44. Omar SA, Adagu IS, Gump DW, Ndaru NP, Warhurst DC. Plasmodium falciparum in Kenya: high prevalence of drug-resistance-associated polymorphisms in hospital admissions with severe malaria in an epidemic area. Ann Trop Med Parasitol. 2001;95:661-9.

45. Staedke SG, Sendagire H, Lamola S, Kamya MR, Dorsey G, Rosenthal PJ. Relationship between age, molecular markers, and response to sulphadoxine-pyrimethamine treatment in Kampala. Uganda. Trop Med Int Health. 2004;9:624-9.

46. WHO. Policy recommendation: intermittent preventive treatment of malaria in pregnancy using sulfadoxine-pyrimethamine (IPTp-SP). Geneva, World Health Organization, 2012.

47. Gutman J, Kalilani L, Taylor S, Zhou Z, Wiegand RE, Thwai KL, et al. The A581G mutation in the gene encoding Plasmodium falciparum dihydropteroate synthetase reduces the effectiveness of sulfadoxine-pyrimethamine preventive therapy in Malawian pregnant women. J Infect Dis. 2015;12:1997-2005. 